\title{
News and Announcements
}

\section{Call for Papers}

\section{"Process Data in Computer-Based Assessment: Opening the Black Box"}

\section{A Special Issue of the European Journal of Psychological Assessment}

\author{
Guest Editors: Marlit Annalena Lindner ${ }^{1}$ and Samuel Greiff ${ }^{2}$ \\ ${ }^{1}$ IPN - Leibniz Institute for Science and Mathematics Education Kiel, Germany \\ ${ }^{2}$ University of Luxembourg, Luxembourg
}

\section{Introduction}

Computer-based assessments have many advantages, one of which is the potential of recording process data that grant closer insights into the interactions of test takers with testing materials and that can be used to increase the quality of psychological assessment. With the growing understanding of the potential of leveraging process data for enhancing both assessment of (i.e., summative assessment) and assessment for learning (i.e., formative assessment) by building on new sources of information, process data are becoming a focus of attention in educational assessment research (e.g., Goldhammer et al., 2014) and beyond.

In this special issue, process data are defined as any behavioral measure that can be repeatedly and automatically logged during a test using technological devices, such as system events and test-taker actions together with their time stamps, but also eye movements, heart rates, or facial expressions. Process measures are not necessarily tied to cognitive outcomes, but could also target affective-emotional states of test takers or their current status of engagement.

Despite great potentials and a growing focus of attention on process data, many challenges remain in terms of integrating the information with test outcome measures and interpreting latent variables in meaningful and valid ways for different students and test situations.

This special issue aims to highlight innovative solutions for integrating different sources of diagnostic information while addressing the challenges researchers and test administrators face when using process data with the goal to ultimately increase test validity and, for example, allow test-takers to best demonstrate their level of achievement.

\section{Submission Process and Timeline}

We encourage interested authors to submit a Letter of Intent (LOI), briefly describing their potential contribution to this special issue, as detailed below.

Contributions may include empirical studies, reports of methodological innovations to combine process data (i.e., logged during testing) with test outcome measures, papers reporting the development and/or validation of new statistical or analytical assessment models based on process data, and theoretical/review papers that focus on the use of process data to advance measures and/or on established psychological theory to offer new perspectives of interpreting potentially ambiguous process measures in the context of psychological assessment. Any of these areas may also require to consider contextual variables (e.g., circumstances of test administration; test content and item formats) and/or individual differences of test takers.

Our indication of topics is not meant to be exhaustive but rather provides general guidance to prospective contributors. This call addresses all areas of psychological research that are involved in the implementation of process data for improving psychological assessment. In addition to educational contexts and performance measures that are one focus area, any contribution that demonstrates innovative 
ways in which process data can be used to improve the quality of psychological assessment are welcome.

However, in line with EJPA's general aim and scope, empirical papers that report the results of studies that are related to process data but do not focus specifically on issues relating to measurement, modeling, or assessment may not be sufficiently relevant.

\section{Letter of Intent}

If you are interested in submitting a manuscript, please send a letter of intent (LOI) to Marlit Annalena Lindner (E-mail mlindner@leibniz-ipn.de), with the subject line "LOI for EJPA special issue" by no later than

\section{July 1, 2021}

LOIs should include a brief (max. 250 words) summary of your proposed contribution, including

- an overview of the topic/question your article will address and

- as much detail as possible of methods and results studies at or near completion are strongly preferred.

This can be in the form of bullet points, an outline, or an abstract, whatever best conveys the proposed content of your article. The LOI should also contain a (tentative) list of authors including institutional affiliations (excluded from the word count).

We expect to communicate decisions on the LOIs to prospective authors by

\section{August 15, 2021}

Only manuscripts that have been invited by the special issue Guest Editors based on the LOI will be considered for inclusion in the special issue.

\section{Authors' Instructions}

Please note that the standards for review maintained by EJPA will be followed and all submissions will be subject to rigorous peer review. An invitation to submit a manuscript does not guarantee that it will ultimately be accepted for the special issue.

Following EJPA's regular instructions for authors, manuscripts submitted for publication in the special issue may be either

(a) Original Articles: no more than 5,000 words (max. 22 pages), or (b) Multistudy Reports: at least two studies conducted with independent datasets, no more than 7,500 words (max. 33 pages), or

(c) Brief Reports: no more than 2,500 words (max. 11 pages).

Page numbers refer to double-spaced, 12-pt font text. The word count includes abstract, text, figures, tables, references, and appendices. Note that Supplementary Material is not included in the word count. Please consult the journal's general guidelines at https://www.hgf.io/ejpa on manuscript submission for more details. Authors are further strongly encouraged to implement open science practices according to the new EJPA Transparency and Openness Promotion Guidelines (Greiff et al., 2020).

Papers acceptable for publication that cannot be published in this special issue may be considered for publication in a regular issue of EJPA, unless authors explicitly decline this option.

\section{Timeline}

Letters of Intent (LOIs) due

Contributing authors confirmed

Authors submit first draft papers to EJPA

Reviewers return comments on manuscripts;

editorial review completed; first decision

communicated to authors

Authors submit revised manuscripts to EJPA

Decision and final comments sent to authors

Final manuscripts due from authors

Special issue finalized

Publication of special issue
July 1, 2021

August 15, 2021

January 15, 2022

April 1, 2022

June 15, 2022

August 1, 2022

October 1, 2022

December 1, 2022

Winter 2022/

Spring 2023

\section{References}

Goldhammer, F., Naumann, J., Stelter, A., Tóth, K., Rölke, H., \& Klieme, E. (2014). The time on task effect in reading and problem solving is moderated by task difficulty and skill: Insights from a computer-based large-scale assessment. Journal of Educational Psychology, 106(3), 608-626. https://doi.org/10.1037/a0034716

Greiff, S., Van der Westhuizen, L., Mund, M., Rauthmann, J. F., \& Wetzel, E. (2020). Introducing new open science practices at EJPA. European Journal of Psychological Assessment, 36(5), 717-720. https://doi.org/10.1027/1015-5759/a000628 